\section{New Zealand - A trainee's paradise?}

Sir: I read with interest Wilkie's briefing on working as a psychiatrist in Australia (Psychiatric Bulletin, 1996, 20,558-560). As an alternative in the Antipodes for British graduates I would suggest considering New Zealand. Temporary registration for three years is open to British and Irish graduates following approval of their documentation. Registered doctors can practise without condition in hospitals or general practice throughout the country.

The rules on immigration depend on the class of visa applied for. A work visa for 12 months requires the offer of a post. An application for a residency visa is more complicated but can be worth the effort involved should you score well on the points system. Maximum points are awarded to professional people between 25 and 29 years of age, speaking English and who have been in full time employment in Europe. A penalty is not incurred for possessing a medical degree as happens in the Australian points system. In New Zealand there is a shortage of trainees in psychiatry. Finding a training post with good teaching in one of the major cities is not difficult.

I spent a 12 month period working in New Zealand where the culture has many similarities to the UK and wonderfully exciting differences. Salaries for doctors give a good standard of living where food and wine especially are cheap. Whether you live in the North or the South Island you are never far from exciting outdoor activities such as skiing in September, sailing at any time or "tramping" in the bush. As an opportunity for training in psychiatry combined with a chance to experience life in a society beyond Europe, there is nowhere more accepting of doctors, nor more agreeable than New Zealand.

\section{K. CoUrtenay, St. George's Hospital, London}

\section{4 hour psychiatric services}

Sir: Rampes \& Sireling (Psychiatric Bulletin October 1996, 20, 622) fear that if informal carers have 24 hour direct access to the psychiatric team this will lead to overloading secondary services with unnecessary contacts and deskilling GPs. In over 20 years of direct experience with carers, I have found views falling into two broad camps over this question. There are those who count themselves 'lucky' who have a 'wonderful' GP, who listens to them, trusts their view of the situation, is willing to make home visits when the patient will not attend the surgery and liaises appropriately with psychiatric and other services. These carers rarely see the need for a 24 hour direct access service. They already have an appropriate and functioning 24 hour service.
Other carers describe their GP variously as 'not interested', 'doesn't care', 'doesn't know anything about schizophrenia'. These are the GPs who do not listen to carer's concerns, who do not understand the pressures and burdens of living with someone with severe mental lllness and who, most tellingly, will only see the patient if he/she attends the surgery. Pleas of 'but he doesn't think there's anything wrong' are dismissed. The patient has to ask to see the doctor, and, since they can walk, must attend surgery. These are the carers who want 24 hour direct access because they have no other way into services. Rather than such a service deskilling GPs it is likely to be seen as necessary because the GP has no skills.

Jacgueline M. ATKinson, Department of Public Health, University of Glasgow, Glasgow G12 8RZ

\section{Case report}

Sir: Henderson \& Reveley (Psychiatric Bulletin, $1996,20,513-515)$ highlight the vulnerability of some women on mixed sex wards and the lack of single sex wards for women wanting this option. The following case illustrates the vulnerability of both sexes on mixed sex wards when single sex facilities are not available.

Case Report. A, a 50-year-old man with a 30 year history of admissions for schizophrenia was admitted to a mixed sex ward. His out-of-hours admission was precipitated by a social crisis and he was free of psychotic symptoms. During the course of his admission an allegation of sexual assault was made against him by B, a 33-year-old female suffering from a first-episode psychosis. This allegation was made a week after the event, was relatively minor, inconsistently described and not witnessed. He denied the allegation and he was not charged after a police interview. He has no previous history of inappropriate sexual behaviour. Subsequently, C made an allegation against $A$ and following police interview, the allegation was deemed to be malicious. Following this second allegation he relapsed and was detained under Section 3 of the 1983 Mental Health Act. He recovered on an increased neuroleptic dose and was discharged. He still remains bitter about his experience. B also recovered and had little recollection of the alleged assault. However, after-care arrangements for both patients were complicated by their residence in the same psychiatric sector with neither of them willing to transfer care to a different sector team. Therefore, arrangements were made to ensure that they did not attend for the same out-patient or day-care session. 